\title{
Spatio-Temporal Prediction Modulates the Perception of Self-Produced Stimuli
}

\author{
Sarah-J. Blakemore \\ Wellcome Department of Cognitive Neurology, University College London \\ Chris D. Frith \\ Wellcome Department of Cognitive Neurology, University College London \\ Daniel M. Wolpert \\ Institute of Neurology, University College London
}

\begin{abstract}
We investigated why self-produced tactile stimulation is perceived as less intense than the same stimulus produced externally. A tactile stimulus on the palm of the right hand was either externally produced, by a robot or self-produced by the subject. In the conditions in which the tactile stimulus was self-produced, subjects moved the arm of a robot with their left hand to produce the tactile stimulus on their right hand via a second robot. Subjects were asked to rate intensity of the tactile sensation and consistently rated self-produced tactile stimuli as less tickly, intense, and pleasant than externally produced tactile stimuli. Using this robotic setup we were able to manipulate the correspondence between the action of the subjects' left hand and the tactile stimulus on their right hand. First, we parametrically varied the delay between the movement of the left hand and the resultant movement of the tactile
\end{abstract}

\section{INTRODUCTION}

Our sensory systems are constantly bombarded by a multitude of sensory stimuli, from which we must extract the few stimuli that correspond to important changes within the environment. One class of stimuli that are in most circumstances unimportant and can be discarded are those that arise as a necessary consequence of our own motor actions. According to several psychological theories, knowledge of our intentions or motor commands is used to distinguish the sensory consequences of our own actions from externally produced sensory stimuli (Decety, 1996; Jeannerod, 1988, 1997; Wolpert 1997; Wolpert, Ghahramani, \& Jordan, 1995). To achieve this, it has been postulated that some kind of central monitor (Frith, 1992) or internal forward model (Wolpert, 1997) can anticipate, and so label correctly, results of intended acts. In particular, the relationship between actions and external events is monitored

to distinguish between events produced by our own actions and events caused by external agents. This can be achieved by capturing the forward or causal relationship between actions, as signalled by "efference copy" (Von Holst, 1954) or "corollary discharge" (Sperry, 1950) and outcomes. By comparing the predicted with the actual sensory feedback, it is possible to distinguish the sensory consequences of our movements from sensory signals due to changes in the outside world.

Von Holst (1954) introduced the idea that when sending motor commands to move the eyes, the motor areas of the brain send a parallel efference copy to the visual areas. This predicts the sensory consequences (corollary discharge) of the movement, and this prediction allows the visual system to compensate for retinal displacement during voluntary eye movements (Sperry, 1950). These mechanisms have mainly been studied with reference to eye movements. However, it appears that sensory predictions produced in conjunction with the motor command 
are not restricted to eye movements but also provide perceptual stability in the context of all self-produced actions: Our ability to monitor, and recognize as our own, self-generated limb movements, touch, and speech suggests the existence of a more general mechanism (Frith, 1992). However, in the oculomotor case complete cancellation is appropriate, whereas in other perceptual systems an efference copy may be used to modulate (not completely cancel) incoming sensory information so that features of importance (those due to external events) are sharpened.

Monitoring self-generated actions allows us to recognize them as our own. Furthermore, evidence suggests that the sensory consequences of some self-generated movements are perceived differently from identical sensory input when it is externally generated. An example of such differential perception is the phenomenon that people cannot tickle themselves (e.g., Claxton, 1975; Weiskrantz, Elliot, \& Darlington, 1971). It has been argued that efference copy produced in parallel with the motor command underlies this phenomenon. In Weiskrantz et al.'s (1971) psychophysical study, a tactile stimulus that transversed the sole of the subject's foot was administered by the experimenter, the subject, or both. Subjects rated the self-administered tactile stimulus as less tickly than the externally administered tactile stimulus. When the stimulation was associated with passive arm movements, tickle strength was reduced but not to the level of the self-administered tactile stimulus. The authors attributed the differences in response to the mode of delivery: Self-administered tactile stimuli produces both an efference copy in accordance with the motor command and reafference produced by the arm movement; passive arm movement produces only reafference, and externally administered tactile stimuli produces neither efference copy or reafference. The authors therefore concluded that although reafference plays a role, the attenuation signal is based mainly on the efference copy signal produced in concordance with self-generated movement.

Claxton (1975) investigated the effect of predictability on tickliness by asking subjects to rate tickliness of a stimulus while being tickled by an external stimulus with their eyes closed or open, and by active and by passive movement. Like Weiskrantz et al. (1971), he found that the presence of corollary discharge during active movement reduced the tickle sensation. In addition he claimed that the more predictable the stimulus, the lower the tickliness rating. However, stimulus predictability was manipulated by having subjects open or shut their eyes. The present study sought to investigate in more detail the conditions under which self-generated tactile stimulation is perceptually attenuated, using a highly predictable tactile stimulus.

By employing a robotic interface we first attempted to replicate Weiskrantz et al.'s (1971) findings by inves- tigating whether tickliness rating is modified by a causative self-generated action. Second, by using two robots so that the tactile stimulus could be delivered under remote control by the subject, we investigated whether tickliness rating is affected by parametrically varied degrees of delay and trajectory perturbation between the movement of the left hand and the tactile stimulus on the right hand. We predicted that increasing the delay and trajectory perturbation would increase the intensity of the tickly sensation because the stimulus would no longer correspond exactly to the efference copy produced in parallel with the motor command. Therefore the actual sensory feedback from the movement would not match the predicted sensory consequences, so attenuation would not occur, and the sensation would become more similar to that caused by an externally administered tactile stimulus (see Figure 1).

Alternatively, self-generated movement could result in a general attenuation of all sensory events occurring simultaneously with the movement. If this second hypothesis were true, no perceptual modulation would be expected to be seen in the delay or trajectory perturbation conditions. This is because under all delays and trajectory perturbations the left hand made the same movement and the right hand experienced the same stimulus. Only the temporal or spatial relationship between the action of the left hand and the sensory effect on the right hand was altered. Any perceptual modulation observed in the delay and trajectory perturbation conditions must be due to a precise attenuation of the sensory feedback, based on specific sensory predictions, rather than a movement-induced nonspecific attenuation of all sensory signals.

One possible reason why subjects in previous studies have rated self-produced tactile sensation as less tickly than externally produced tactile sensation is that in the former case subjects know exactly when and where the next touch will occur. Perhaps tickle requires an element of surprise. Previous studies have attempted to control for this by making the tactile stimulus as predictable as possible. However, none used robots to control the tactile stimulus precisely. In the present study we employed robots to produce the tactile stimulation, so the stimulus was highly controlled and highly predictable in time and space in the externally produced tactile sensation condition. Therefore lack of stimulus predictability could be ruled out as a factor responsible for increasing tickliness rating in this condition. Furthermore, to avoid biasing subjects toward tickling and minimizing the likelihood of them using their tacit knowledge about tickling oneself, the experiment was not framed as pertaining to tickling or ticklishness per se. Instead we referred to the experiment as concerning "tactile sensation" and asked subjects to rate the stimulus in terms of several sensations, including tickliness (painful, intense, pleasant, irritating, and tickly). 
Figure 1. A model for determining the sensory consequences of a movement, in the present study, whether the left hand is causing the tactile sensation on the right hand. An internal forward model makes predictions of the sensory feedback based on the motor commands sent to the left hand. These predictions are then compared to the actual sensory feedback to produce the sensory prediction errors. The lower the errors, the greater is the attenuation of tactile sensation. In our study, when there is no delay or trajectory perturbation, the model correctly predicts the sensory consequences of the movement, so no error ensues between the predicted and actual sensory information. In this case the motor command to the left hand can be used to attenuate predictively the sensation on

the right hand. As the sensory feedback deviates from the prediction of the model (by increasing the delay or trajectory perturbation), the error between predicted and actual sensory feedback increases. This leads to a decrease in the amount of attenuation possible and a relative increase in the intensity of sensation experienced on the right hand.

\section{RESULTS}

After practice each subject was able to produce the desired movement of the left hand with reasonable accuracy. Painful received zero ratings in all conditions by all subjects and was therefore excluded from the analysis. Irritating received very few ratings, so was also excluded from the analysis.

\section{Self-Produced Versus Externally Produced Tactile Stimulation}

Subjects rated the self-administered tactile stimulus as significantly less Tickly $(F=41.21 ; p<0.0001)$, Intense $(F=112.948 ; p<0.0001)$, and Pleasant $(F=60.157 ; p<$ $0.0001)$ than the externally administered tactile stimulus. Figure 2 shows Tickly rating ranks for self-produced and externally produced tactile stimulation.

\section{Delays}

Two subjects reported noticing the delay in the delay conditions in the postexperiment debriefing. The other 14 subjects claimed to be unaware of the delays. There was a significant increase in Tickly $(F=24.93 ; p<$ $0.0005)$ rating as the delay between the movement of the left hand and the tactile stimulus increased from 0 to $200 \mathrm{msec}$. There was no significant difference be- tween ratings at 200- and 300-msec delay and in the externally produced tactile stimuli condition. Figure 3 shows Tickly rating ranks with increasing delay between the movement of the left hand and tactile stimulus on right hand.

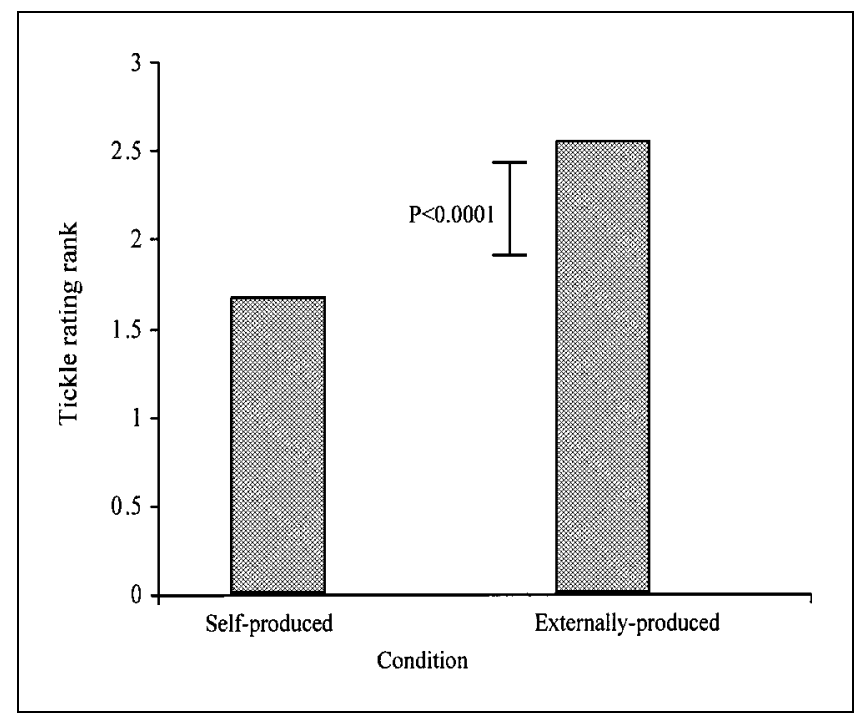

Figure 2. Graph to show Tickly rating ranks for self-produced and externally produced tactile stimulation conditions (all subjects combined). There was a significant difference between ratings for these two conditions. 
Figure 3. Graph to show Tickly rating ranks with increasing delay between action of left hand and tactile stimulus on right hand (all subjects combined). Ratings increased significantly from 0 - to 100 msec, and 100- to 200-msec delay.

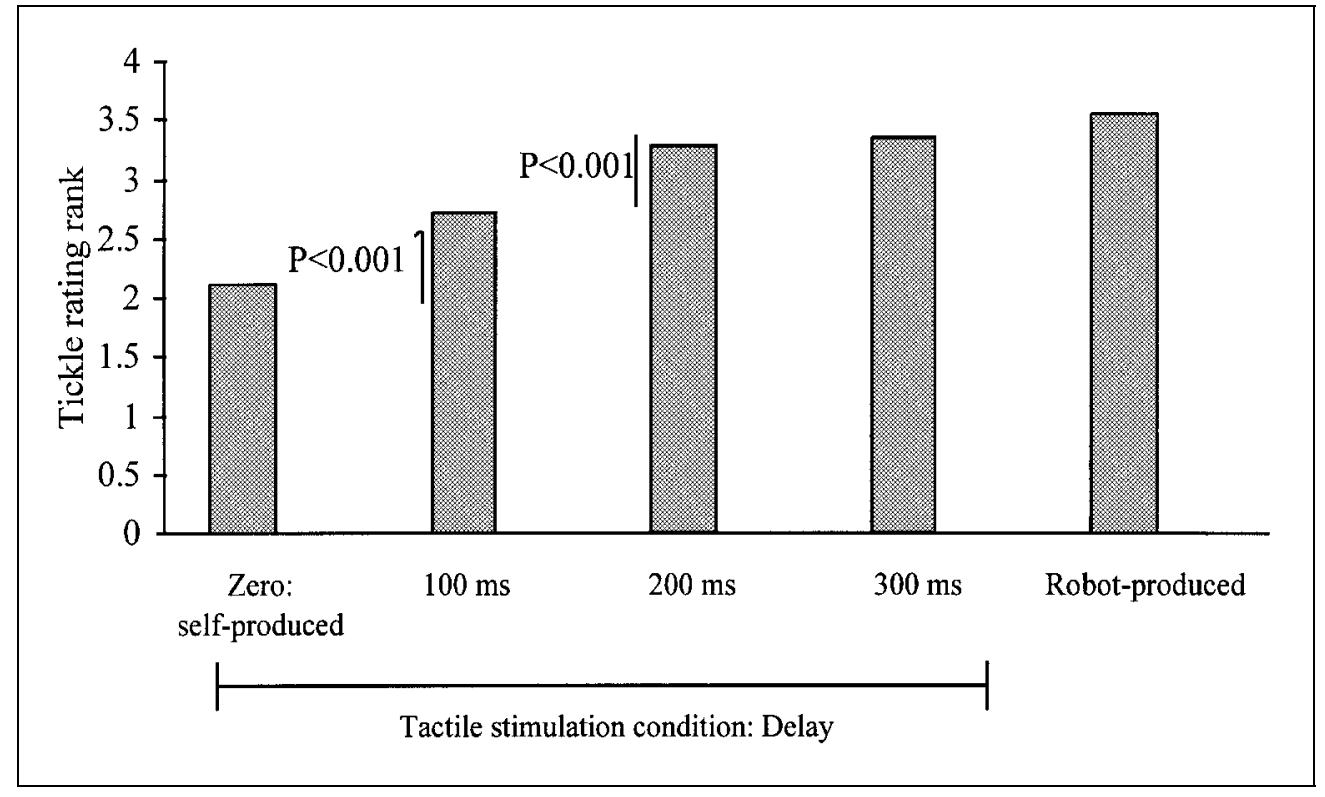

\section{Trajectory Perturbations}

There was a significant increase in Tickly $(F=9.37 ; p<$ 0.01 ) rating as trajectory perturbation between movement and tactile stimulation increased from 0 to $90^{\circ}$. There was no significant difference between ratings at $90^{\circ}$ and in the externally produced tactile stimuli condition. Figure 4 shows that the Tickly rating ranks with increasing trajectory perturbation between the movement of the left hand and tactile stimulus on right hand.

\section{Difference Between Rating Scales}

As a measure of the precision with which subjects could rate the different percepts, we calculated the variance of the rating of each percept for each subject and condi- tion. The average variance across all subjects and conditions was significantly higher for Pleasant $(0.40 \pm 0.005)$ and Intense $(0.46 \pm 0.006)$ than for Tickly $(0.26 \pm 0.012)$ ratings.

\section{DISCUSSION}

The present study sought to investigate how subjects perceive the sensory consequences of their own actions and under what conditions this perception changes. We demonstrate that tickliness rating is modulated by a causative self-generated action: Subjects consistently rated a self-produced tactile sensation as being less tickly than when it was externally produced. Furthermore, tickliness rating increased with increasing degrees of delay and trajectory perturbation between the actions of the
Figure 4. Graph to show Tickly rating ranks with increasing trajectory perturbation between action of left hand and tactile stimulus on right hand (all subjects combined). Ratings increased significantly from 0 to $30^{\circ}$ and from 60 to $90^{\circ}$ trajectory perturbation.

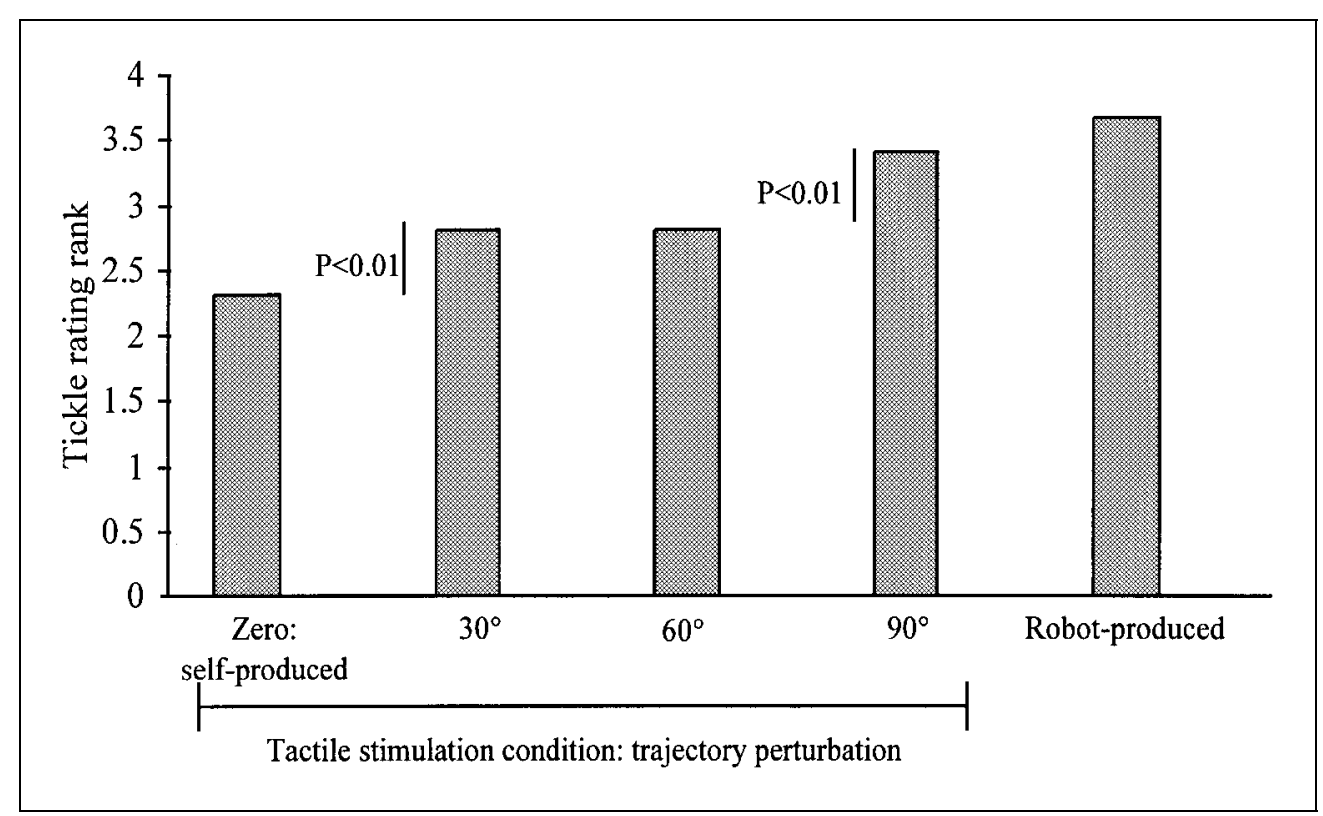


left hand and the resultant tactile stimulus on the right hand. We argue that increasing the delay and trajectory perturbation increases the intensity of the sensation because the stimulus no longer corresponds (in time or space) to the motor command, and hence the efference copy cannot accurately predict or compensate for the sensory consequences of the action.

Our results support and expand on work by Weiskrantz et al. (1971), demonstrating that self-produced tactile sensations are consistently rated as less tickly than externally produced tactile sensations. In addition, we demonstrate that tactile sensation becomes more tickly the further the tactile stimulus diverges from the motor command producing it. By introducing delays and trajectory perturbations between the action of the left hand and the resultant tactile stimulus on the right hand, we demonstrate that increases in these two parameters produces corresponding increases in tickliness rating. In contrast, although there was a trend, there was no correlation between the degree of delay or trajectory perturbation and the Intense and Pleasant ratings. This might be due to the higher variance of ratings shown by subjects on these measures compared to the tickliness rating. This lower consistency for Intense and Pleasant suggests that subjects found assessing and quantifying these sensations more difficult than the tickliness of a stimulus.

It might be argued that the greater the delay or trajectory perturbation between the active and passive hands, the less predictable the stimulus becomes, and that decreasing predictability is responsible for increasing Tickly ratings. However, in the delay and trajectory perturbation conditions the stimulus was still predictable in the sense that the stimulus on the right hand is entirely determined by the movement of the left hand. Furthermore, it was possible for subjects to learn after a few seconds the exact relationship between the action of their left hand and the stimulus on their right hand. Indeed in the debriefing following the experiment only two subjects reported having noticed the presence of any kind of anomaly in the delay conditions. Thus most subjects were unaware of the delay and the tactile stimulus was as expected in these conditions as in the zerodelay condition. In those conditions in which trajectory perturbations were used, subjects were aware of them (their right hand experienced lateral tactile stimulation, whereas their left hand moved in a different direction), but the tactile stimulus became predictable after the first few seconds, in which it was possible to learn this relationship. A more plausible explanation than stimulus predictability is that as the tactile stimulus diverges temporally or spatially from the motor command producing it, the efference copy is less able to predict precisely and cancel the sensation.

Under all delays in the current study the left hand made the same movement and the right hand experienced the same stimulus. Only the temporal relationship between the action of the left hand and the sensory effect on the right hand was altered. The results suggest that the perceptual attenuation of self-produced tactile stimulation is due to a precise modulation of the sensory feedback, based on specific sensory predictions, rather than a nonspecific movement-related attenuation of all sensory signals. If this latter hypothesis were true, no perceptual modulation would be expected to be seen in the delay or trajectory perturbation conditions.

The ability to anticipate the sensory consequences of our own actions is possible using a forward model of the motor system. Forward models capture the forward or causal relationship between actions and outcomes by using an efference copy of the motor command. A computational mechanism by which the attenuation of selfproduced tactile sensation might be achieved is in terms of the sensory prediction errors made by a forward model (see Figure 1; Wolpert, 1997).

In the case of the present experiment, based on the efference copy produced in parallel with the motor command, the forward model predicts the sensory consequences of the movement of the left hand. These sensory predictions are compared to the actual sensory feedback from the movement and are used to cancel the tactile consequences of the movement. The closer the system is to direct contact between the hands, the more of the sensory reafference will be cancelled, as demonstrated by the present results. When there is no delay or trajectory perturbation, the model correctly predicts the sensory consequences of the movement, so no error ensues between the predicted and actual sensory information. In this case the motor command to the left hand can be used to cancel predictively the sensation on the right hand. As the sensory feedback deviates from the prediction of the model (by increasing the delay or trajectory perturbation), the error between predicted and actual sensory feedback increases. This leads to a decrease in the amount of attenuation possible and a relative increase in the intensity of sensation experienced on the right hand. The present results demonstrate that Tickly rating is minimal with zero delay or trajectory perturbation and increases smoothly as the (imperceivable) delay or the trajectory perturbation increases up to a certain point $\left(200 \mathrm{msec}\right.$ or $90^{\circ}$, respectively), at which stage the sensation becomes indistinguishable from an externally produced sensation. This suggests that a precise forward model is in operation that is sensitive even to small changes in the correspondence, including those unavailable to conscious awareness.

Unlike in the oculomotor system, complete cancellation of incoming sensory information may not be appropriate for the somatosensory system because it would result in anaesthesia during self-produced movements. We propose that prediction-based modulation acts as a filter on incoming sensory signals that can enhance the 
afference-to-reafference ratio (akin to increasing the signal-to-noise ratio). This modulation of incoming sensory input might have the effect of accentuating features of importance (for example, those due to external events). In addition the oculomotor comparator operates only on direct commands to move the eyes. When the eye is moved by pressing on the eyeball with a finger, the percept is that the world moves. However, this sort of interaction is rare in everyday life, and there would be little advantage of it being modeled by the central nervous system. In contrast, interactions between other body parts such as the limbs are common, and for such interactions we have shown that predictions of the sensory consequences of the motion of one hand are used to attenuate the sensation on the other hand.

The attenuation of the perception of self-produced stimuli is well documented in humans (Angel \& Malenka, 1982; Chapman, Bushnell, Miron, Duncan, \& Lund, 1987; Collins, Cameron, Gillard, \& Prochazka, 1998; Milne, Aniss, Kay, \& Gandevia, 1988). Possible physiological mechanisms by which this attenuation of self-produced tactile stimuli is mediated have been postulated on the basis of research on animals. Neurophysiological data demonstrate that neuronal responses in the somatosensory cortex are attenuated by self-generated movement (see Chapman, 1994, for review). For example, active touch is "gated" in the somatosensory cortex of rats (Chapin \& Woodward, 1982) and monkeys (Chapman \& AgeraniotiBelanger, 1991; Jiang, Chapman, \& Lamarre, 1991) compared to passive and external touch of an identical tactile stimulus. In addition, functional imaging (fMRI) data from human subjects demonstrate an increase in activity of the primary and secondary somatosensory cortex when subjects experienced an externally produced tactile stimulus on their palm relative to a self-produced tactile stimulus (Blakemore, Wolpert, \& Frith, 1998a, 1998b). We propose that this inhibition of somatosensory cortex activity by self-generated movements could result from the comparison between predicted and actual sensory feedback, which results in no error when a tactile stimulus is self-produced. There is evidence that this comparison might take place in the cerebellum (Ito, 1970; Leiner, Leiner, \& Dow, 1995; Miall, Weir, Wopert, \& Stein, 1993; Paulin, 1989; Wolpert, Miall, \& Kawato, 1998), a proposal supported by the functional imaging data.

What is the purpose of this perceptual attenuation of self-produced tactile stimuli? First, externally produced stimuli normally carry more biological significance than self-produced stimuli and self-produced stimuli need not be picked out as important. An animal must be attuned to sensory events that indicate the actions of other animals, and this can only be achieved by being able to ignore the sensory events that arise as a consequence of the animal's own actions. This allows unexpected stimulation to be selectively detected. The attenuation of self-produced tactile stimuli might distinguish them from biologically more important (externally produced) stimuli.

Second, the nature of tickliness has been the center of much debate, mainly with reference to tickle-induced laughter. The debate is split between those who argue that tickle-induced laughter is purely reflexive (Fridlund \& Loftis, 1990; Harris and Christenfeld, 1997; Stearns, 1972; Sully, 1902) and those who propose that it is socially induced and results from close physical contact with another person (Darwin, 1872; Hecker, 1873; McDougall, 1922). The inability to tickle oneself has been taken to support the latter theory (Gregory, 1924; Hoshikawa, 1991; Shultz, 1976). It has been argued that tickle-induced laughter depends on a second person doing the tickling (Levine, 1979) and as such has been termed a purely psychological process (McDougall, 1922): The person's cognitions about the situation determine whether laughter occurs or not (Darwin, 1872; Foot \& Chapman, 1976; Hecker, 1873; Koestler, 1964). However, our findings demonstrate that the tickliness of a tactile stimulus is proportional to the sensory errors in prediction made by a forward model based on the motor command producing the stimulus. This suggests that the function of the attenuation of self-produced tactile sensations might be to differentiate self-produced from externally produced tactile sensations. We suggest that one purpose of this differentiation is to prevent laughter in the former case. It is not merely the tactile stimulation that induces tickle-induced laughter; laughter only occurs if the tactile stimulus is tickly, which requires it to be externally produced. In this case the stimulus is not physiologically gated, which signals that someone else is producing it.

\section{CONCLUSION}

In conclusion, our study suggests that an internal forward model is able to provide information about the motor commands sent to one hand, which is used to cancel the resultant tactile sensation in the other hand. We argue that the tickliness of a tactile stimulus is proportional to the error produced by the comparison between the predicted sensory consequences of a motor command and the actual sensory feedback from the movement. As the tactile stimulus diverges temporally or spatially from the motor command producing it, the efference copy is less able to predict and cancel the sensation, which is therefore perceived as more tickly.

\section{METHODS}

\section{Subjects}

A total of 16 normal right-handed subjects (age range 19 to 30 years), who were naive to the issues involved in the research, gave their informed consent and partici- 
pated in the present study, which was approved by the National Hospital for Neurology and Neurosurgery Ethics Committee.

\section{Apparatus}

Subjects sat at a table with their right forearm laid on the table with the palm facing upward. A tactile stimulus constituting a piece of foam (radius, $1 \mathrm{~cm}$ and length, 2.5 $\mathrm{cm}$ ) attached to the end of a lightweight, robotic manipulator (Phantom Haptic Interface, Sensable Devices, Cambridge, MA) was positioned above their right palm. The robot was controlled by a personal computer which was capable of generating forces in three dimensions, and its position was updated at $1 \mathrm{kHz}$. The subject gripped a cylindrical object (radius, $1 \mathrm{~cm}$ and width, 4 $\mathrm{cm}$ ) with the thumb and index finger of their left hand. This object was held directly above the tactile stimulus and was attached to a second robotic device (see Figure 5). In each trial of each condition the tactile stimulus on the right palm moved sinusoidally at a frequency of $2 \mathrm{~Hz}$

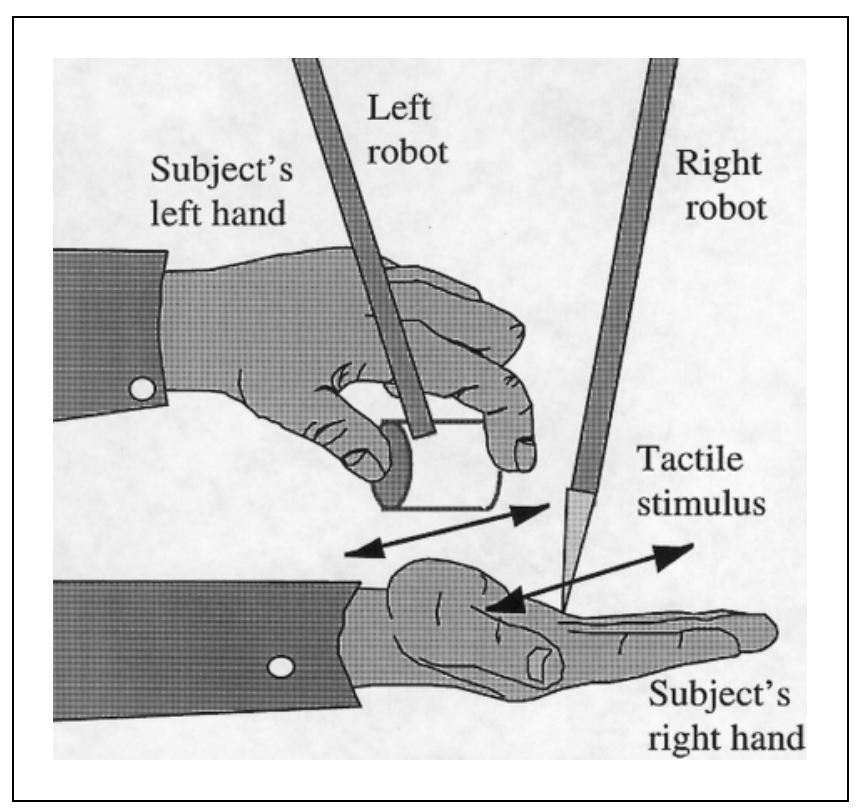

Figure 5. Diagram of experimental setup. A tactile stimulus constituting a piece of foam attached to the end of a robotic manipulator was positioned above the subjects' right palm. The subjects gripped a cylindrical object with the thumb and index finger of their left hand. This object was held directly above the tactile stimulus and was attached to a second robotic device. In the externally produced tactile stimulus condition the right robot was programmed to produce the sinusoidal tactile stimulus movement on the subjects' right hand. In all the self-produced tactile stimulus conditions subjects were required to move the object held in their left hand sinusoidally, which, via two robots, produced the same movement of the tactile stimulus above their right hand. Delays and trajectory perturbations could be introduced between the movement made by the left hand and the resultant movement of the right robot. See Methods for details. and amplitude $1.5 \mathrm{~cm}$ in the lateral direction, with a downward force of $0.5 \mathrm{~N}$.

In the self-produced tactile stimulus conditions subjects were required to move the object held in their left hand sinusoidally (frequency, $2 \mathrm{~Hz}$ and amplitude, 1.5 $\mathrm{cm}$ ), which, via two robots, produced the same movement of the tactile stimulus above their right hand. Subjects practiced this until they were proficient at producing the desired frequency and amplitude of movement. This took between 30 and 60 sec for each subject. In the conditions in which the movement of the tactile stimulus above their right hand was produced by the movements of their left hand, subjects were told of this relationship.

Trials consisted of two 10-sec subtrials. Two points were marked on the subjects' right palm, and subjects were instructed (by a tone) to move their hand from one point to the other during the 3-sec delay between the two subtrials. Subjects shut their eyes throughout the experiment. The conditions were randomized and counterbalanced within and between subjects, and subjects were blind to the order.

In the externally produced tactile stimulus condition the right robot was programmed to produce the sinusoidal tactile stimulus on the subjects' right hand. With their left thumb and index finger subjects gripped the left robot, which remained still throughout.

In the self-produced tactile stimulus condition subjects were required to move the object in their left hand sinusoidally at $2 \mathrm{~Hz}$ to the desired amplitude. This movement was faithfully transmitted to the tactile stimulus via the right robot, which produced the same trajectory, frequency, and amplitude of movement above the subject's right palm.

In the delay conditions 100-, 200-, and 300-msec delays were introduced between the movement made by the left hand and the resultant movement of the right robot. Subjects were not informed of the presence of these delays.

In the trajectory perturbation conditions perturbations of 30,60 , and $90^{\circ}$ rotations were introduced between the direction of movement made by the left hand and the direction of movement of the right robot. The tactile stimulus always moved in the same (lateral) direction on the palm of the subject's right hand, but subjects were instructed to move the left hand along different trajectories. Straight lines drawn on paper corresponding to 30,60 , and $90^{\circ}$ rotations from the lateral direction indicated to the subjects the direction in which they should move their left hand in each condition.

\section{Rating Scale}

Subjects were instructed to rate the sensation on their palm on a scale from 0 (not at all) to 10 (extremely) intense, painful, tickly, pleasant, and irritating. They 
were told that it was important to maintain the same scale all the way through the experiment and that the emphasis was on the relative values of their judgments, not the absolute values.

\section{Data Analysis}

Because subjects used individual subjective rating scales, the ratings do not conform to a bivariate normal distribution. To test the significance of association between the variables we ranked the variances and calculated the coefficient of rank correlation. Friedman's nonparametric ranking test was used to rank the ratings and to calculate the coefficient of rank correlation, in the following conditions:

Self-produced tactile stimulation and externally produced tactile stimulation

Self-produced tactile stimulation, delays 100,200, and 300 msec, and externally produced tactile stimulation

Self-produced tactile stimulation, trajectory perturbations 30,60 , and $90^{\circ}$ and externally produced tactile stimulation.

A repeated measures multivariate analysis of variance (MANOVA) was performed to assess the effects of selfgenerated movement, delays, and trajectory perturbations on the ratings. Results were taken as significant if $p<0.05$.

\section{Acknowledgements}

We thank the referees and James Kilner for useful comments. This work was supported by the Wellcome Trust. SJB is supported by a Wellcome Trust 4-year Ph.D. program in Neuroscience at University College London.

Reprint requests should be sent to Sarah-Jayne Blakemore, Wellcome Department of Cognitive Neurology, Institute of Neurology, University College London, 12 Queen Square, London WC1N 3BG, UK, or via e-mail: s.blakemore@ucl.ac.uk.

\section{REFERENCES}

Angel, R. W., \& Malenka, R. C. (1982). Velocity-dependent suppression of cutaneous sensitivity during movement. Experimental Neurology, 77, 266-274.

Blakemore, S.-J., Wolpert, D. M., \& Frith, C. D. (1998a). Why can't we tickle ourselves? An fMRI study. Neuroimage 7, 481.

Blakemore, S.-J., Wolpert, D. M., \& Frith, C. D. (1998b). Central cancellation of self-produced tickle sensation. Nature Neuroscience, 1, 635-640.

Chapin, J. K., \& Woodward, D. J. (1982). Somatic sensory transmission to the cortex during movement: Gating of single cell responses to touch. Experimental Neurology, 78, 654-669.

Chapman, C. E. (1994). Active versus passive touch: Factors influencing the transmission of somatosensory signals to primary somatosensory cortex. Canadian Journal of Physiological Pharmacology, 72, 558-570.

Chapman, C. E., \& Ageranioti-Belanger, S. A. (1991). Compari- son of the discharge of primary somatosensory cortical (SI) neurones during active and passive tactile discrimination. Proceedings, Third IBRO World Congress of Neuroscience, August 4-9, Montreal, Quebec, 317.

Chapman, C. E., Bushnell, M. C., Miron, D., Duncan, G. H., \& Lund, J. P. (1987). Sensory perception during movement in man. Experimental Brain Research, 68, 516-524.

Claxton, G. (1975). Why can't we tickle ourselves? Perceptual and Motor Skills, 41, 335-338.

Collins, D. F., Cameron, T., Gillard, D. M., \& Prochazka, A. (1998). Muscular sense is attenuated when humans move. Journal of Physiology, 508, 635-643.

Darwin, C. (1872). The expressions of the emotions in man and animals. London: John Murray.

Decety, J. (1996). Neural representation for action. Reviews in the Neurosciences, 7, 285-297.

Foot, H. C., \& Chapman, A. J. (1976). The social responsiveness of young children in humorous situations. In A. J. Chapman \& H. C. Foot (Eds.), Humor and laughter: Theory, research, and applications (pp. 187-214). London: Wiley.

Fridlund, A. J., \& Loftis, J. M. (1990). Relations between tickling and humorous laughter: Preliminary support for the Darwin-Hecker hypothesis. Biological Psychology, 30, 141150.

Frith, C. D. (1992). The cognitive neuropsychology of schizophrenia. London: Erlbaum.

Gregory, J. C. (1924). The nature of laughter. London: KeganPaul.

Harris, C. R., \& Christenfeld, N. (1997). Humor, tickle and the limitations of the Darwin-Hecker hypothesis. Cognition and Emotion, 11, 103-110.

Hecker, E. (1873). Die physiologie and psychologie des lachen und des komischen. Berlin: F. Dummler.

Hoshikawa, T. (1991). Effects of attention and expectation on tickle sensation. Perceptual and Motor Skills, 72, 27-33.

Ito, M. (1970). Neurophysiological aspects of the cerebellar motor control system. International Journal of Neurology, 7, 162-176.

Jeannerod, M. (1988). The neural and behaviorial organization of goal-directed movements. Oxford, UK: Oxford University Press.

Jeannerod, M. (1997). The cognitive neuropsychology of action. Cambridge, UK: Blackwell.

Jiang, W., Chapman, C. E., \& Lamarre, Y. (1991). Modulation of the cutaneous responsiveness of neurones in the primary somatosensory cortex during conditioned arm movements in the monkey. Experimental Brain Research, 84, 342354.

Koestler, A. (1964). The act of creation. London: Hutchinson.

Leiner, H. C., Leiner, A. L., \& Dow, R. S. (1995). The underestimated cerebellum. Human Brain Mapping, 2, 244-254.

Levine, J. (1979). Humor and psychopathology. In C. Izard (Ed.), Emotions in personality and psychopatbology (pp. 37-69). New York: Plenum.

McDougall, W. (1922). Why do we laugh? Scribners, 71, 359363.

Miall, R. C., Weir, D. J., Wolpert, D. M., \& Stein, J. F. (1993). Is the cerebellum a Smith predictor? Journal of Motor Behavior, 25, 203-216.

Milne, R. J., Aniss, A. M., Kay, N. E., \& Gandevia, S. C. (1988). Reduction in perceived intensity of cutaneous stimuli during movement: A quantitative study. Experimental Brain Research, 70, 569-576.

Paulin, M. G. (1989). A Kalman filter theory of the cerebellum. In E. M. A. Arbib \& E. S. Amari (Eds.), Dynamic interactions in neural networks: Models and data (pp. 241-259). Springer-Verlag. 
Shultz, T. R. (1976). A cognitive-developmental analysis of humor. In A. J. Chapman \& H. C. Foot (Eds.), Humor and laughter: Theory, research, and applications (pp. 12136). London: Wiley.

Sperry, R. W. (1950). Neural basis of spontaneous optokinetic responses produced by visual inversion. Journal of Computational Physiology and Psychology, 43, 482-489.

Stearns, F. R. (1972). Laughing. Springfield, IL: Charles C. Thomas.

Sully, J. (1902). An essay on laughter. New York: Longmans, Green.

Von Holst, E. (1954). Relations between the central nervous system and the peripheral organs. The British Journal of Animal Behavior, 2, 89-94.

Weiskrantz, L., Elliot, J., \& Darlington, C. (1971). Preliminary observations of tickling oneself. Nature, 230, 598-599.

Wolpert, D. M. (1997). Computational approaches to motor control. Trends in Cognitive Sciences, 1, 209-216.

Wolpert, D. M., Ghahramani, Z., \& Jordan, M. I. (1995). An internal model for sensorimotor integration. Science, 269, 1880-1882.

Wolpert, D. M., Miall, R. C., \& Kawato, M. (1998). Internal models in the cerebellum. Trends in Cognitive Sciences, 2, 338-347. 